\title{
Vortex chip incorporating an orthogonal turn for size-based isolation of circulating cells
}

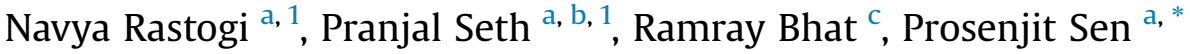 \\ a Centre for Nano Science and Engineering, Indian Institute of Science, Bangalore, 560012, India \\ ${ }^{\mathrm{b}}$ Department of Biomedical Engineering, McGill University, Montreal, H3A OG4, Canada \\ ${ }^{\mathrm{c}}$ Department of Molecular Reproduction, Development and Genetics, Indian Institute of Science, Bangalore, 560012, India
}

\section{H I G H L I G H T S}

- Orthogonal vortex chip for label-free size-specific cell and particle isolation.

- Orthogonal configuration enhances formation of vortices and engenders turn-effect.

- Inertial forces involved in turn-effect result in differential ejection of particles.

- Vortex-trapping is accomplished at lower flow velocities which aids cell viability.

- Results affirm applicability of device in gentle isolation of circulating tumor cells.

\section{A R T I C L E I N F O}

\section{Article history:}

Received 15 September 2020

Received in revised form

23 February 2021

Accepted 15 March 2021

Available online 22 March 2021

\section{Keywords:}

Microfluidics

Particle separation

Cell separation

Vortex-trapping

Inertial microfluidics

Non-linear microfluidics

\section{G R A P H I C A L A B S T R A C T}

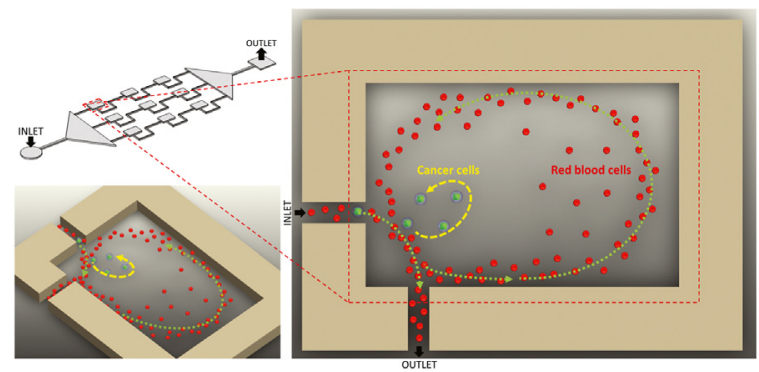

\begin{abstract}
A B S T R A C T
Size-based label-free separation of rare cells such as CTCs is attractive due to its wider applicability, simpler sample preparation, faster turnaround and better efficiency. Amongst such methods, vortextrapping based techniques offer high throughput but operate at high flow velocities where the resulting hydrodynamic shear stress is likely to damage cells and compromise their viability for subsequent assays. We present here an orthogonal vortex chip which can carry out size-differentiated trapping at significantly lower (38\% of previously reported) velocities. Composed of entry-exit channels that couple orthogonally to a trapping chamber, fluid flow in such configuration results in formation of a vortex which selectively traps larger particles above a critical velocity while smaller particles get ejected with the flow. We call this phenomenon the turn-effect. Critical velocities and optimal architectures for trapping of cells and particles of different sizes are characterized. We explain how shear-gradient lift, centrifugal and Dean flow drag forces contribute to the turn-effect by pushing particles into specific vortex orbits in a size- and velocity-dependent fashion. Selective trapping of human breast cancer cells mixed with whole blood at low concentration is demonstrated. The device shows promising results for gentle isolation of rare cells from blood.
\end{abstract}

() 2021 Elsevier B.V. All rights reserved.

\footnotetext{
* Corresponding author. Indian Institute of Science, Bangalore, Centre for Nano Science and Engineering, Bangalore, Karnataka, 560012, India.

E-mail addresses: navyarastogi@iisc.ac.in (N. Rastogi), pranjal.seth@mail.mcgill.ca (P. Seth), ramray@iisc.ac.in (R. Bhat), prosenjits@iisc.ac.in (P. Sen).

1 P. Seth and N. Rastogi contributed equally to this work.
} 


\section{Introduction}

The isolation of a specific cellular niche from heterogeneous populations has been an enduring topic of intense investigation at the interface of biology and microfluidics [1-7]. This is especially relevant in the context of the separation of circulating tumor cells (CTCs) from the blood of patients afflicted with cancer. CTC detection can be used as a diagnostic assay [8], a method to determine the efficacy of treatment [9] and to indicate the relapse of cancer $[10,11]$. The ability to culture the captured patient-derived CTCs [12] would allow us to further investigate disease progression and therapeutic outcomes in greater detail and on a personalized level [13].

Existing technologies carry out separation by either inducing external forces (active separation) or by using intrinsic hydrodynamic forces (passive separation). Active separation methods include dielectrophoresis [14], magnetophoresis [15], acoustophoresis [16], etc., where the flow direction of the selected particles is modified using an external force. Drag-induced primary motion of particles is diverted by the external forces being invoked, which can precisely control cells of interest and can be adjusted in real time. However, these external forces are typically in the order of $\mathrm{pN}$, resulting in a low sample processing rate with volume flow of about $100 \mu \mathrm{L} / \mathrm{min}$ and particle translation speed in the range of $100 \mu \mathrm{m} / \mathrm{s}$. This ultimately yields a limited throughput. Moreover, these techniques often involve biomarkers [17-22] and labeling agents [23] which may be incompatible with strategies for further culture of these cells and clinical applications. Additionally, active separation strategies often fail to isolate the entire population of target cells [24] due to the inherent heterogeneity in the expression of markers and membrane proteins which are often the ligands for labeling agents and enriching methodologies [25,26].

Passive separation on the other hand is carried out by simply controlling the hydrodynamic properties of flow. These include pinched flow fractionation [27], Dean flow fractionation [28], deterministic lateral displacement [29], microfiltration [30], inertial microfluidics [31-33], etc. Though somewhat non-specific in identifying CTCs based on their biochemical characteristics, these techniques incorporating flows and geometries that are inventive and conspicuous, are able to separate and concentrate cells based on the difference in their inherent physical properties such as size, deformability, etc. The fixed geometry and restriction in design of passive microdevices limit their range of operation for different samples [34]. In addition, passive label-free separation may ensure sensitive separation, but specificity will be less than their affinitybased counterparts. Nonetheless, passive separation techniques can be easily parallelized to harness their respective advantages. Inertial microfluidics in particular provides a high-throughput, whereby large sample volumes can be processed within a short time interval. Operating at flow rates of up to $5 \mathrm{~mL} / \mathrm{min}$, this advantage is especially useful for processing high volumes of bodily fluids such as blood and ascites for tumor cells. Owing to the rarity of CTCs, the capability to quickly process large volumes is a practical necessity. Shorter processing times are also known to result in lower death rates of CTCs which enhances their usability for future assays $[35,36]$.

In the flow regime where the inertial effects become pronounced, a separating Stokes flow is seen for flows around bodies placed in an uniform flow or flows in a channel with sudden expansion [37]. These are known as Moffat eddies [38], which grow into fully developed microscale laminar vortices as the Reynolds number of the flow increases. Such vortices combined with inertial focusing at sufficiently high Reynolds number have been strategically utilized by Di Carlo and coworkers to carry out size-selective isolation of cells $[24,32,39,40]$. However, these vortex-trapping techniques are able to operate only at high flow-velocities of up to $4 \mathrm{~m} / \mathrm{s}$, where the resulting hydrodynamic shear stress is likely to damage the cells and compromise their viability [41-43] for downstream applications.

In this paper, we describe the design and characteristics of a passive and continuous microfluidic device that is capable of sizebased trapping and separation of rare cells from a heterogeneous suspension. The aim of the new design is to achieve vortextrapping at lower flow velocities. Fig. 1A shows a generalized schematic for this vortex chip with orthogonal arrangements of entry and exit for fluid flow. Within the trapping chamber, configuration-specific formation and growth of microscale-vortex with flow velocity is observed. Above a critical velocity, the vortex selectively entraps larger beads and cells, since they are ejected out of the flow owing to the 'turn effect'. This turn effect is due to a combination of physical forces arising in the fluid flow trajectory due to the sharp turn resulting from the orthogonal entry-exit configuration. Meanwhile, smaller red blood cells predominantly escape the trapping chamber with the flow, thus enabling separation of the rare larger cells. This device operates at flow-velocities that are up to $38 \%$ of the previously reported vortex-trapping methods [24,32,39,40,44,45], which should improve the viability of cells for culture-based applications. Further, lower velocities imply lower operational pressures, which simplifies device fabrication and operation. Exploiting some of the unique features of inertial $[46,47]$ and non-linear [48] microfluidics, we present here a simple and practical size-differentiated separation technique which could have broad applicability.

\section{Materials and methods}

\subsection{Sample preparation}

Polystyrene beads of size $15 \mu \mathrm{m}, 20 \mu \mathrm{m}(10.1 \% \mathrm{w} / \mathrm{w})$ were purchased from Bangs Laboratories Inc. These were used to prepare particle sample solutions by diluting the particle suspensions in PBS or DI water, along with $0.1 \% \mathrm{v} / \mathrm{v}$ Tween-80 (Sigma-Aldrich) solution added to prevent particle aggregation. The mixture was mixed vigorously using a vortexer. High concentration beads solution was used for experiments with CCD camera, consisting of bead concentration $1 \% \mathrm{v} / \mathrm{v}$ in DI water. For experiments with highspeed camera, solution containing bead concentration $0.1 \% \mathrm{v} / \mathrm{v}$ in $1 \mathrm{X}$ PBS, along with $1.66 \% \mathrm{v} / \mathrm{v}$ whole blood was used. Low particle concentration experiments were done with $0.001 \% \mathrm{v} / \mathrm{v} 20 \mu \mathrm{m}$ beads and 30 MDA-MB-231 cells/mL. Cancer cell experiments were carried out with High-speed camera, with sample solution consisting of cancer cells (MDA-MB-231, MCF-7 and BT549) concentration $\sim 8 \times 10^{4}$ cells $/ \mathrm{ml}$ in $1 \mathrm{X}$ PBS along with $1.66 \% \mathrm{v} / \mathrm{v}$ whole blood.

\subsection{Experimental setup}

Microparticle/cell samples were injected into the device from a plastic syringe using a New Era syringe pump (NE-8000). Owing to the high pressure and high flow rates involved, connectors were custom made using catheters, infant-feeding tubes, micropipette tips, and epoxy adhesive. (See Fig. S1) Imaging was carried out using either a CCD camera (IDS Imaging UI-3240CP-C) with a 3X lens, or a high-speed camera (FASTCAM Mini UX100, Photron) at $6400 \mathrm{fps}$ coupled to microscope with 10X magnification lens. MATLAB R2019a was used to generate scatter plots through image processing techniques. 

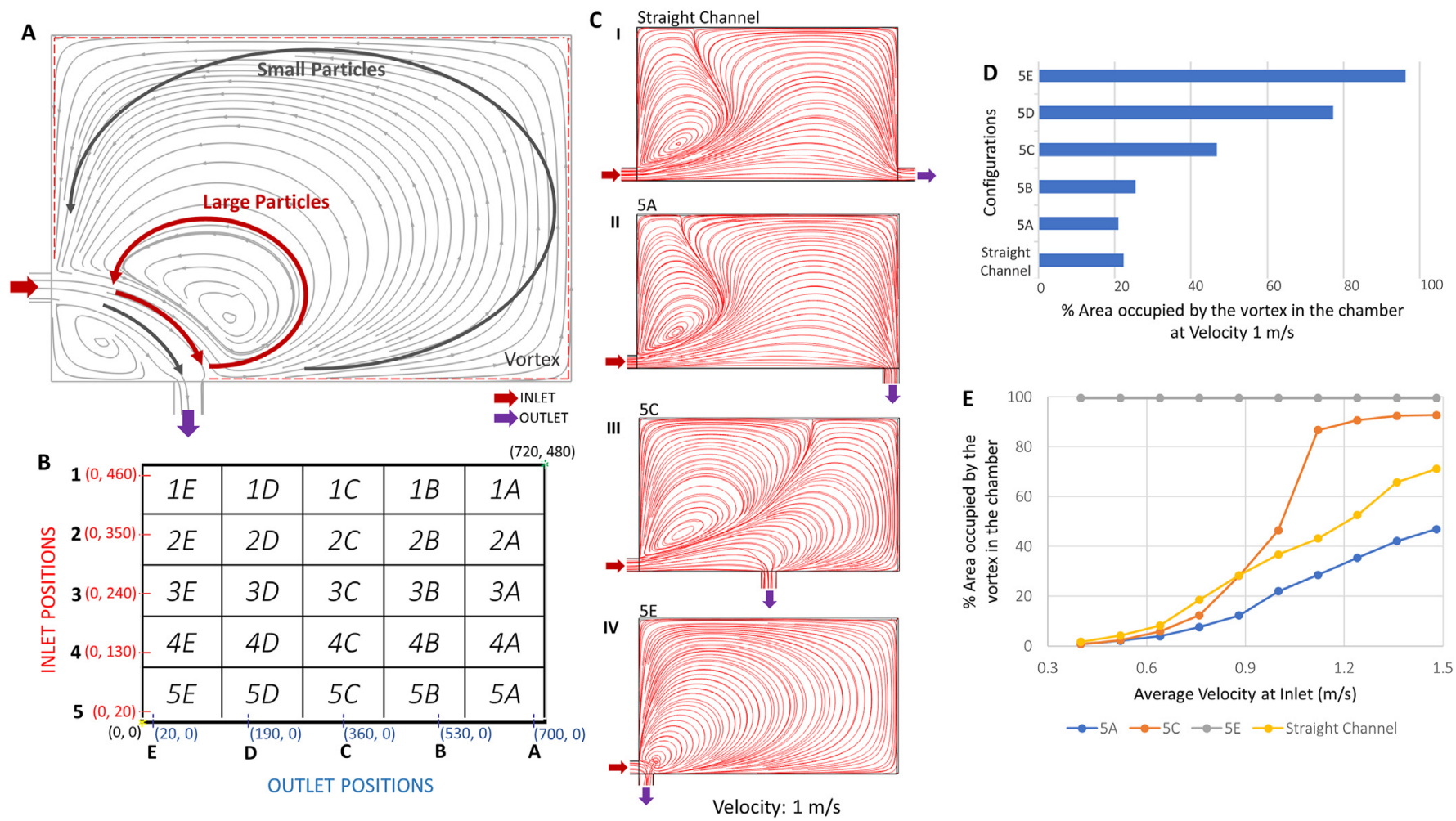

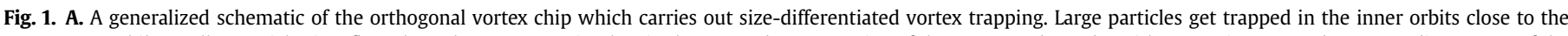

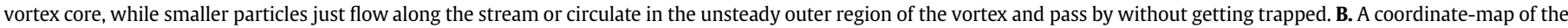

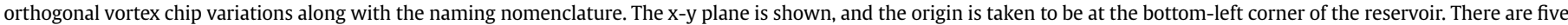

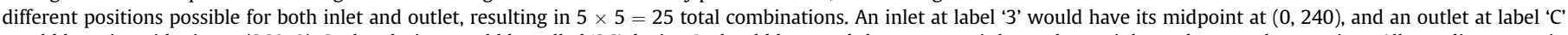

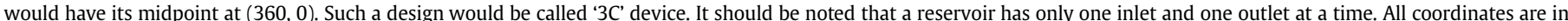

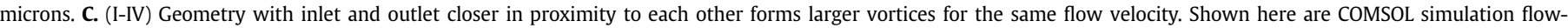

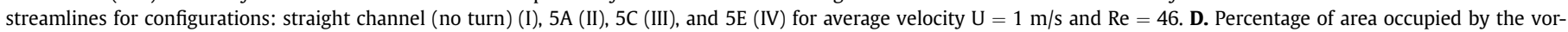

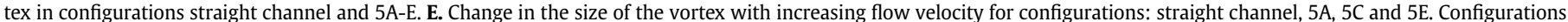
with an outlet located closer to the inlet exhibit saturation with a fully developed vortex that occupies most of the reservoir area at lower flow velocities.

\subsection{Live dead analysis}

A solution of MDA-MB-231 $\left(3 \times 10^{6}\right.$ cells/ml) in PBS was used to perform the live dead experiment. The suspension was injected through the 3E design of 4 channels with 6 reservoirs each at an average inlet velocity of $1.3 \mathrm{~m} / \mathrm{s}$ and collected. $0.4 \%$ Trypan blue (Himedia Laboratories Pvt. Ltd) was used in a dilution of 1:1 to count the live and dead cells in a Neubauer's hemocytometer.

\section{Results and discussion}

\subsection{Simulation results}

Previous efforts at vortex based size-specific cell trapping utilized straight flows at high Reynolds numbers [24,32,39,40,44,45]. We began by asking whether introducing an orthogonal bend in the flow trajectory might allow formation of a large vortex suitable for stably trapping particles and cells at lower flow rates. We used COMSOL Multiphysics to simulate fluid flows through such designs. 3D flow simulations were carried out and Navier-Stokes equations for incompressible flow were utilized in the Laminar Flow module. No-slip boundary condition was used at the walls and zero pressure boundary condition was used at the outlet. Flow conditions were varied by changing the average flow velocity at the inlet (See Fig. S2). The trapping chamber dimension was kept the same $(480 \mu \mathrm{m} \times 720 \mu \mathrm{m})$ as in previous efforts by other groups $[24,32,39,40]$ in order to allow comparison with those results.
Several distinct variations of this orthogonal configuration based on the location of inlet and outlet were studied. Fig. 1B provides a detailed description of the nomenclature system used for identifying device geometries. Flow simulations were performed for these configurations with the objective of generating maximally sized vortices at the lowest flow velocities. Fig. 1C shows flow streamlines from COMSOL simulations for selected configurations suggesting that as the inlet and outlet are brought closer in proximity to each other, the area covered by the vortex increases for the same average flow velocity of $1 \mathrm{~m} / \mathrm{s}$. The percentage area covered by the vortex for these configurations was quantified using Image J and is shown in Fig. 1D. Configurations with outlet placed closer to inlet are observed to yield a larger vortex. Fig. 1E shows the change in area occupied by the vortex in these geometries for increasing flow velocity. Configurations with inlet and outlet placed closer to each other are observed to attain a saturated state, where the vortex occupies most of the reservoir area, at lower flow velocities. The simulations therefore indicate that certain orthogonal flow designs produce larger vortices at lower velocities.

\subsection{Flow and trapping characterization}

Next, we sought to experimentally verify our modeling results. We further wanted to characterize the minimum velocity required for trapping particles of a given size. Microfluidic chips were made using standard cleanroom fabrication and soft lithography techniques as detailed in Supplementary Methods. Briefly, a silicon 
wafer substrate coated with a positive photoresist was selectively UV-exposed, developed, and etched to obtain a master-mold. Liquid PDMS was casted on this prepared mold, peeled off after curing, and was plasma-bonded to glass slide to obtain microfluidic devices. A suspension of microparticles (polystyrene beads of $12 \mu \mathrm{m}$ and $15 \mu \mathrm{m}$ diameter) was flowed in these microfluidic devices in order to visualize the flow streams, verify the presence of the vortices, study their shape, characterize the velocities required for trapping and examine the particle behavior.

We observed that the trajectory of particles in these microparticle-laden flows depends on both particle sizes and flow velocities, as shown in Fig. 2A for design 4C. At lower velocities, the particles follow the streamlines and exit the chamber with the flow. For instance, $20 \mu \mathrm{m}$ and $15 \mu \mathrm{m}$ beads do not get trapped up to $1.12 \mathrm{~m} / \mathrm{s}$ and $1.24 \mathrm{~m} / \mathrm{s}$ respectively. Above a critical velocity, the particles start escaping the flow and get trapped in the vortex, which then appears as an enclosed recirculating trajectory. We define this minimum flow velocity at which particles of a given size get trapped in the vortex as the trapping-velocity; and the recirculating path itself is defined as the trapping-orbital. Experimentally observed trapping-velocity for $20 \mu \mathrm{m}$ and $15 \mu \mathrm{m}$ beads in design $4 \mathrm{C}$ is $1.24 \mathrm{~m} / \mathrm{s}$ and $1.36 \mathrm{~m} / \mathrm{s}$ respectively. Increasing flow velocity further also reveals multiple stable orbits for $15 \mu \mathrm{m}$ beads at $1.36 \mathrm{~m} /$ s. Thus, for a fixed flow velocity, different-sized particles exhibit their own unique trajectories in the reservoir with larger particles getting trapped at lower velocities. Moreover, while COMSOL simulations confirm the formation of vortex even at low velocities, vortex trapping is observed only above certain flow velocities which is specific for a given particle size. This implies that vortex formation by itself does not necessarily lead to trapping of particles and a nuanced effect of inertial forces is at work here.

For each inlet-outlet configuration, a different trapping velocity specific to each particle size was observed. Trapping-velocity for
$15 \mu \mathrm{m}$ and $20 \mu \mathrm{m}$ beads was experimentally determined for all inlet-outlet configurations as shown in the 2-dimensional colorcoded map in Fig. 2B. Velocity- and size-specific behavior pertaining to trapping-velocity described above for design $4 \mathrm{C}$ was observed to be consistent for other inlet and outlet configurations as well. In general, as the inlet and outlet are brought close to each other, we observe that the trapping velocity decreases. This is expected as the turn-radius decreases when the inlet and outlet come closer. Turn-radius is defined as the effective radius of the circular path taken by flow at the turn (see Fig. S3(A)). As explained in section 3.4 describing the turn effect, a smaller turn-radius enhances the inertial effects responsible for the ejection of particles from the flow stream.

However, the distribution of trapping velocity in Fig. 2B is neither monotonic nor symmetric. For example, we observe that for the closest inlet-outlet configuration (design 5E), the trapping velocity for the $20 \mu \mathrm{m}$ beads is higher than the neighboring configurations. This is possibly due to the extremely short path of the bead in the trapping chamber. Due to the shorter flow path within the chamber, larger inertial forces are required to obtain the requisite deflection for the particle to leave the flow stream. Further, at the velocities used in these studies, the flow does not follow the shortest path between the inlet and outlet for some of the designs, rather it takes an arched trajectory (as seen in Fig. S3(A)). Hence, the turn-radius has a non-trivial relationship with respect to inlet-outlet position. We believe that these are the underlying reasons for the observed deviations in the distribution of trapping velocity.

Finally, for the same flow velocity and bead size, a variation in the inlet-outlet configuration also led to a change in the size and position of the trapping-orbital around the vortex (Fig. S4). Considering steric effects, a purely geometric argument implies that the number of beads that could be trapped within a vortex also
A

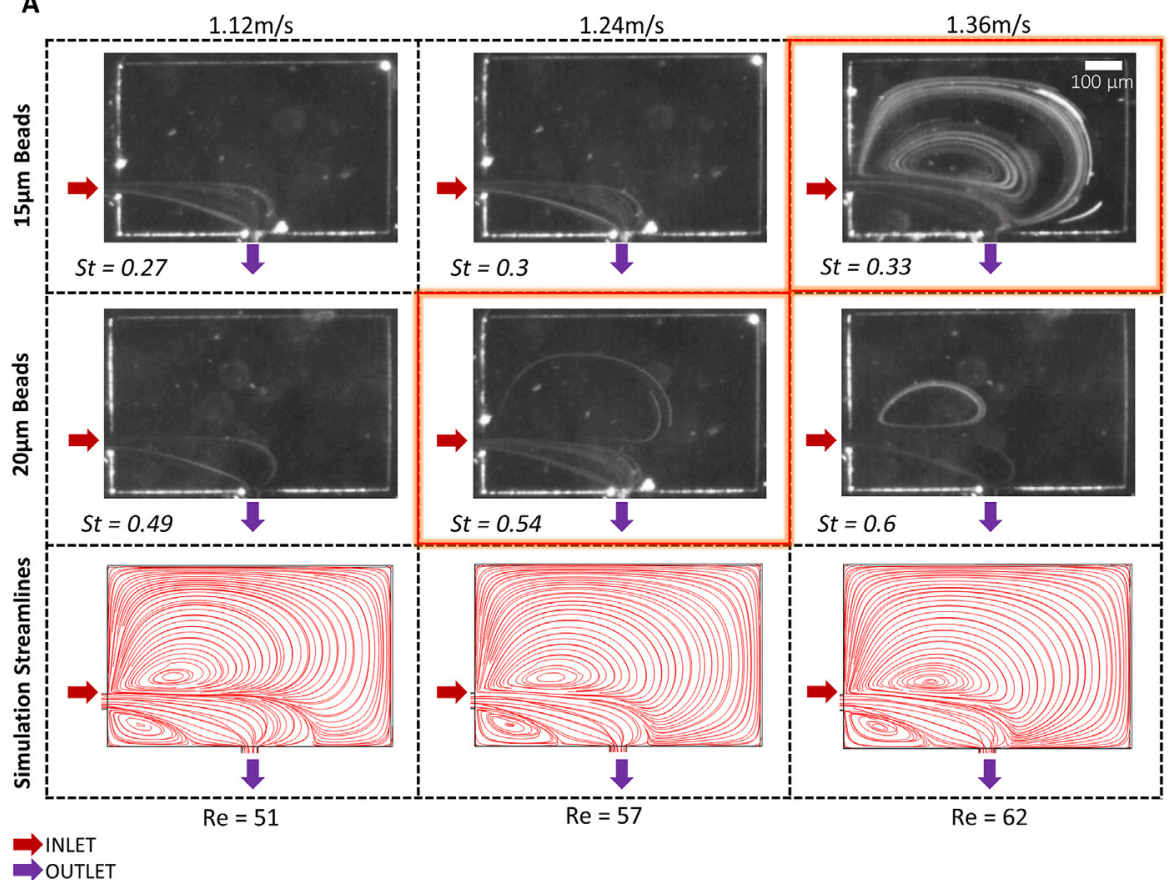

B

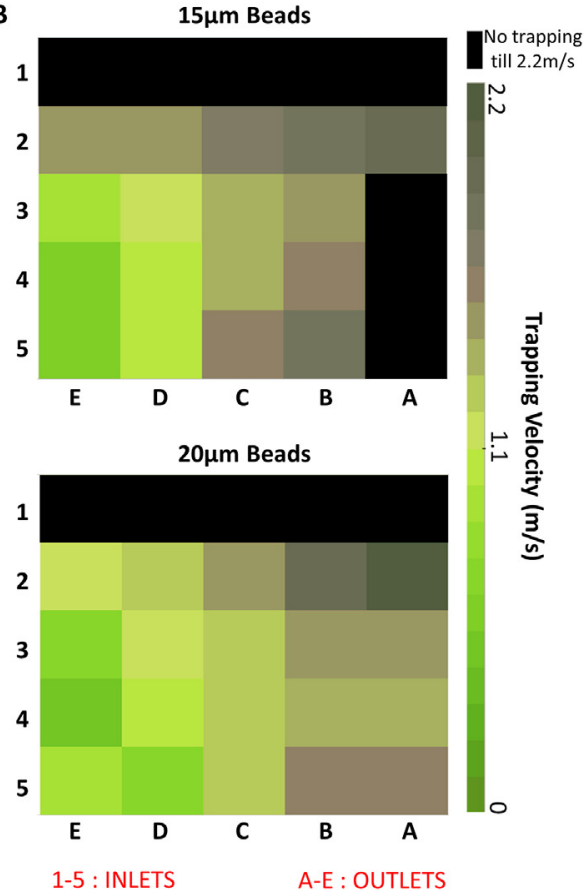

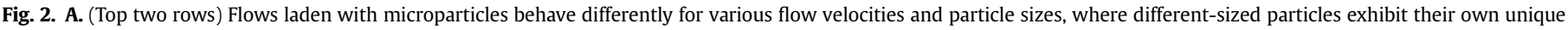

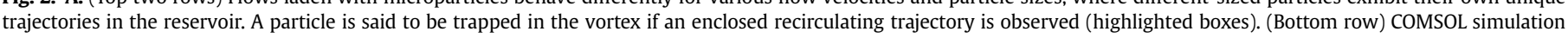

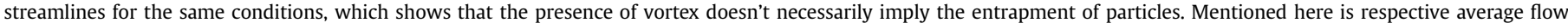

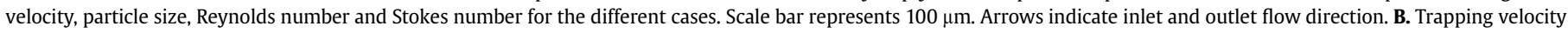
of particles ( $15 \mu \mathrm{m}$ and $20 \mu \mathrm{m}$ ) for all the 25 different inlet-outlet designs. 
changes with the inlet-outlet configuration. Even though trapping occurs at lower velocities for the closer inlet-outlet configurations, the resulting trapping-orbitals were small. Steric effects should limit the number of particles that can occupy these orbitals. Larger trapping-orbitals were observed when the distance between the inlet and outlet was increased. These larger orbitals should be able to accommodate a greater number of particles before the steric effects start to play a role. However, at the same time, placing the inlet and outlet far apart required higher trapping velocity and resulted in unsteady trapping. This unsteady trapping is characterized by two observations. Firstly, the particles are observed to leave the main flow stream and enter the chamber. However, they are unable to get into a steady orbit and exit the chamber without getting trapped. Secondly, in cases where the particles are trapped, one or more particles in the trapping-orbital were observed to leave the trap and exit the chamber after some time, possibly due to small flow rate fluctuations (see Fig. S3(A)). These collective observations advocate that an optimum zone should exist whereby stable trapping could be accomplished at low velocities. Hence, out of the 25 possible configurations, designs $3 \mathrm{D}, 3 \mathrm{E}, 4 \mathrm{D}$ and $4 \mathrm{E}$ were chosen (Fig. S3(B)) for further studies as these exhibited stable particle trapping at the lowest possible velocities. The suitability of these designs is addressed again in section 3.4 describing the turn effect.
We also conducted high-speed videographic studies to observe the trapped particles in these four designs that were deemed optimal. A suspension of particles with reduced concentration was used in order to observe individual particle behavior in the reservoir. A steady entrapment was seen in most cases where a few larger rare particles got trapped in the reservoir, but there were instances where the particles already trapped in the vortex would get 'knocked out' (See Fig. 3A and Movie M1) due to a new incoming particle. Few particles were also seen to enter and exit the vortex area without getting trapped in an occupied orbital. Both of the above observations suggest that steric effects play a role in limiting the number of particles that every trapping-orbital can accommodate. The trapping-orbitals serve as "attractor-zones", where they exhibit steady entrapment for the particles. Separate stable orbitals would exist corresponding to every particle size at a given flow rate. Also, multiple attractor-zones or trapping-orbitals may coexist within the vortex as seen in Fig. $2 \mathrm{~A}$ for $15 \mu \mathrm{m}$ beads at flow velocity $1.36 \mathrm{~m} / \mathrm{s}$. Particles were also observed to be trapped in recirculating trajectories in different vertical planes (Fig. 3A(i)). This might be due to the existence of distinct flow profiles in planes at different depths (Fig. S5) in the reservoir because of which multiple stable orbitals are possible.
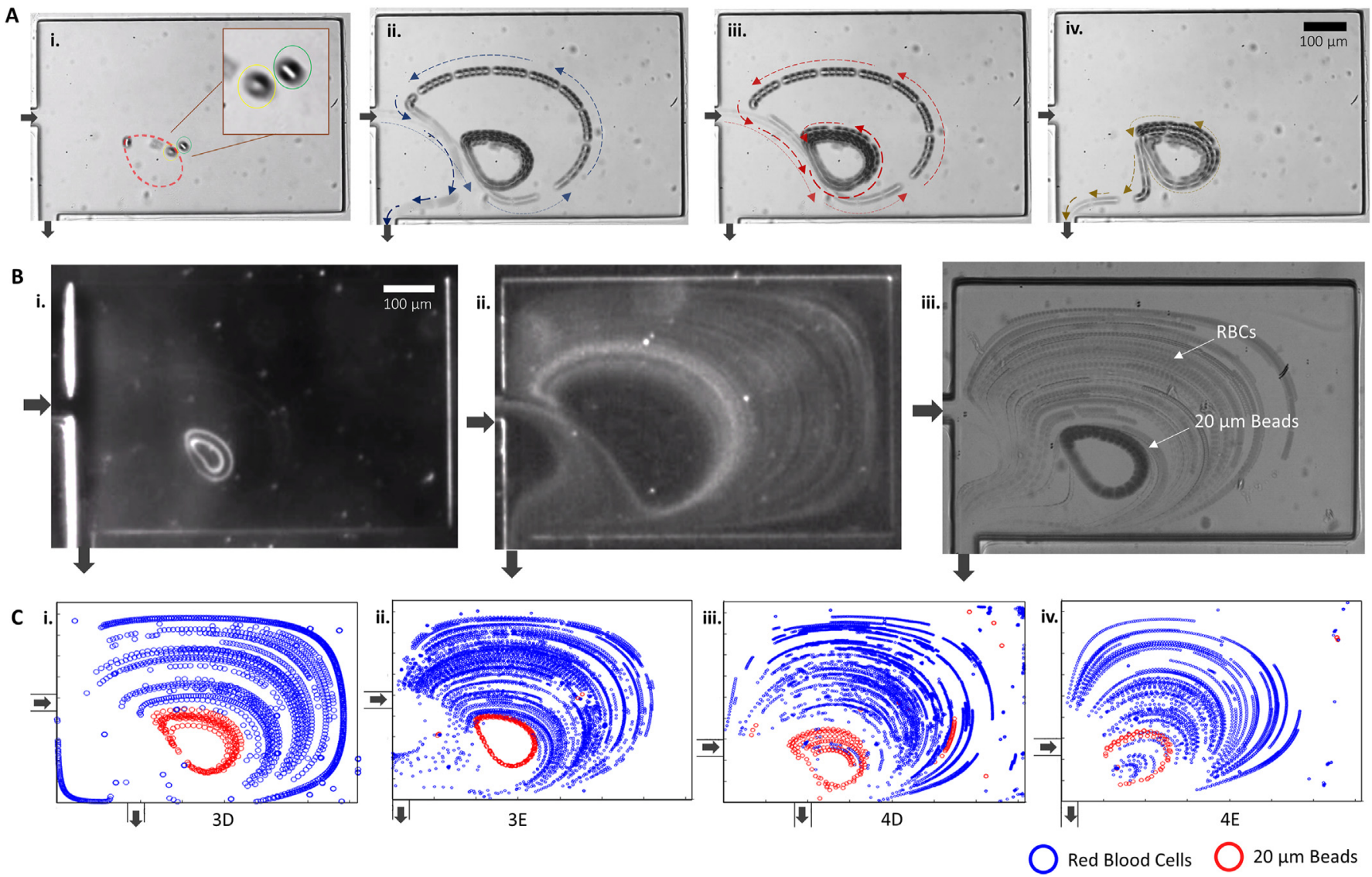

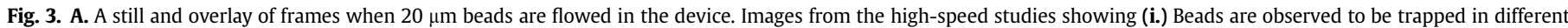

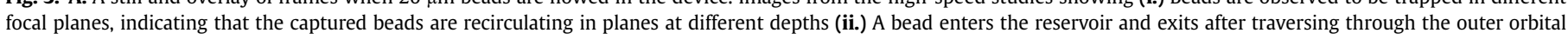

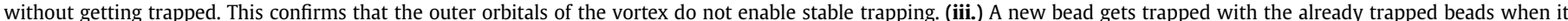

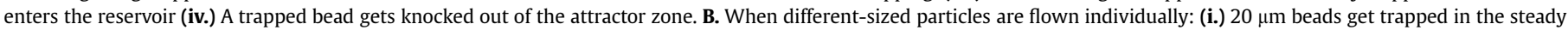

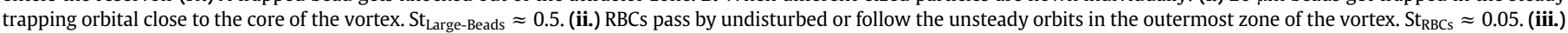

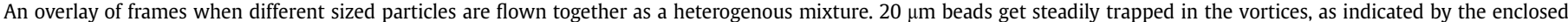

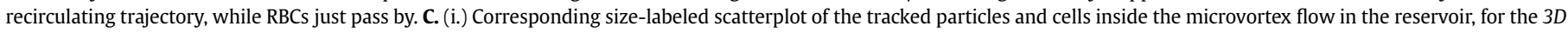
design. Similar scatter plots are obtained for (ii.) $3 E$ (iii.) $4 D$ (iv.) $4 E$. Scale bar represents $100 \mu \mathrm{m}$. All flows are at $\mathrm{U}=1.3 \mathrm{~m} / \mathrm{s}$. 


\subsection{Trapping induced separation of cells/particles}

We next asked whether beads of large sizes would continue to get trapped within vortices upon flowing in a heterogeneous suspension that also consisted of red blood cells (RBCs) as smaller particles. As discussed before, particle-particle or particle-cell interaction cannot be neglected. Hence, there is always a possibility that larger but rare particles in the trapping-orbital are 'knocked-out' by one of the numerous smaller cells. Successful trapping under this scenario necessitates that the trapping-orbital of the larger particle is distinct from the path taken by the smaller cells (RBCs).

To understand the trapping behavior, beads and diluted whole blood were flowed separately at different velocities. Above a critical velocity, the larger beads $(20 \mu \mathrm{m})$ were trapped in the stable orbitals close to the vortex core as seen in Fig. $3 \mathrm{~B}(\mathrm{i})$. On the other hand, blood cells when flowed at the same velocity through the same design were observed to exit it without entering the vortex, or merely exited after traversing the outermost orbitals of the vortex as seen in Fig. 3B(ii). This exclusivity of the trapping-orbital and the path taken by RBCs provided a flow regime where trappinginduced separation or concentration enhancement would be possible.

When heterogeneous samples consisting of both blood cells and beads were flowed together (see Methods for details of sample preparation), they retained their individual behaviors (Fig. 3B(iii) and Movie M2). This was also confirmed through the scatterplots generated using the high-speed camera videos, capturing the flow of heterogenous mixture of $20 \mu \mathrm{m}$ beads and RBCs (Fig. 3C). Earlier reports have confirmed that the outermost region of microfluidic vortices tend to be unstable allowing particles to easily escape the vortex, whereas particles get stably trapped in the innermost orbits [44]. This allows us to execute a vortex-trapping induced segregation of larger particles or cells. Once isolated and trapped in the vortex, the larger particles can be collected by switching to a buffer solution flowed at low velocity. The trapped larger particles thus get flushed out and are collected, thereby completing the separation process. We sought to explain the phenomenon of size-specific entrapment and localization within vortices in the reservoir with orthogonal inlet and outlet by investigating the forces acting on the particle and measuring its Stokes number.

\subsection{Turn-effect}

The observed turn-effect is a result of multiple inertial forces being exerted on the particle in flow. In this section, we discuss these forces and their individual contribution to the phenomenon. A schematic representation of all the forces is shown in Fig. 4A. While flowing in the channel, particles experience hydrodynamic inertial focusing as they travel downstream, being subjected to a shear-gradient lift force directed towards the channel wall and an opposing wall-effect lift force directed towards the channel centerline [49]. Using scaling arguments, the resultant lift force has been described as [50]:

$F_{L i f t}=f_{L}\left(x, R e_{c h}\right) \rho_{f} U^{2} \frac{a^{3}}{D_{h}}$

where $\rho_{f}$ is the density of fluid, $a$ is the particle diameter, $U$ is the flow velocity, $f_{L}$ is the lift coefficient which is a function of particle's lateral position in the channel $x$ and channel Reynolds number $R e_{c h}$, and $D_{h}$ is the hydraulic diameter of the channel defined in terms of width $W$ and height $H$ as $D_{h}=2 W H /(W+H)$. When this focused particle enters the reservoir, the neighboring microchannel wall is no longer in its vicinity and the hydrodynamic interaction with the wall disappears [47]. In the absence of the wall-lift force, the remaining shear-gradient lift pushes the particle away from the centerline flow stream directing it down the shear-gradient towards the center of the trapping vortex [45]. (See Fig. S6.)

Another effect arises due to the higher density of the particles in comparison to the surrounding fluid. The particle experiences centrifugal force due to the curvilinear trajectory which imparts a transverse motion away from the centerline flow stream towards the trapping vortex. This centrifugal force on the particle can be written as [51]:

$F_{\text {Centrifugal }}=\frac{\left(\rho_{P}-\rho_{f}\right) \pi a^{3} U_{P}^{2}}{6 r_{\text {turn }}}$

where $\rho_{P}$ is the density of the particle, $U_{P}$ is the particle velocity, $r_{\text {turn }}$ is the turn-radius of the particle's curvilinear trajectory (see Fig. S3(A)). This force increases with reduction of the turn-radius $\left(F_{\text {Centrifugal }} \propto 1 / r_{\text {turn }}\right)$.

The curvilinear path of the streamlines also gives rise to transverse Dean flows [52,53]. Fig. 4A(ii) and 4A (iii) show the secondary flows in the channel cross-section. It is important to note that unlike curved channels, here the curvilinear flow path is not bounded by the channel walls. Hence a single cross-section does not capture the transverse plane for flow at different positions. We are however able to observe the plane of inflection. On the left of the plane of inflection the transverse flows drag the particle towards the outlet. However, on the right, the particles are strongly dragged towards the vortex. Secondary flow results in an additional transverse drag force on the particles, which for conventional curved channels has been stated as [54]:

$F_{\text {Dean }}=5.4 \times 10^{-4} \pi \eta a\left(\sqrt{\frac{D_{h}}{r_{\text {turn }}}} R_{c h}\right)^{1.63}$

where $\eta$ is the dynamic viscosity. The Dean drag arising here from the curvilinear flow further aids in transverse particle migration out of the centerline flow stream into the vortices. Like centrifugal force, Dean drag force also increases with decrease in the turn radius $\left(F_{\text {Dean }} \propto 1 / \sqrt{r_{\text {turn }}}\right)$. Lift force, however, is independent of the turn-radius and hence should not change with inlet-outlet configuration. Lift force (equation (1)) and centrifugal force (equation (2)) both are directly proportional to the cubic power of the particle diameter $\left(\propto a^{3}\right)$. In contrast to the other forces, Dean drag is proportional to particle diameter $(\propto a)$ and hence changes more slowly with change in particle size.

The cumulative result of all the forces described above is a transverse migration force that has a complex dependency on the inlet-outlet configuration, particle diameter and velocity. In addition to these forces, the particle also experiences a drag force due to the primary flow from the inlet to the outlet:

$F_{\text {drag }}=3 \pi \eta a U$

Acting in the direction of the primary flow, this drag force carries the particle along with it. Hence, the ejection of particle from the primary flow and its trapping in the vortex is the result of a competition between the primary drag and the transverse migration forces (shear-gradient lift, centrifugal and Dean drag). For a fixed particle size, at lower flow velocities, the primary drag dominates and the particles follow the flow. As the flow velocity is increased, the transverse forces increase faster than the primary drag due to $U^{2}$ dependence. For a given flow condition, these forces are much more pronounced for the larger $20 \mu \mathrm{m}$ particles compared to the smaller RBCs owing to its larger diameter. Larger 

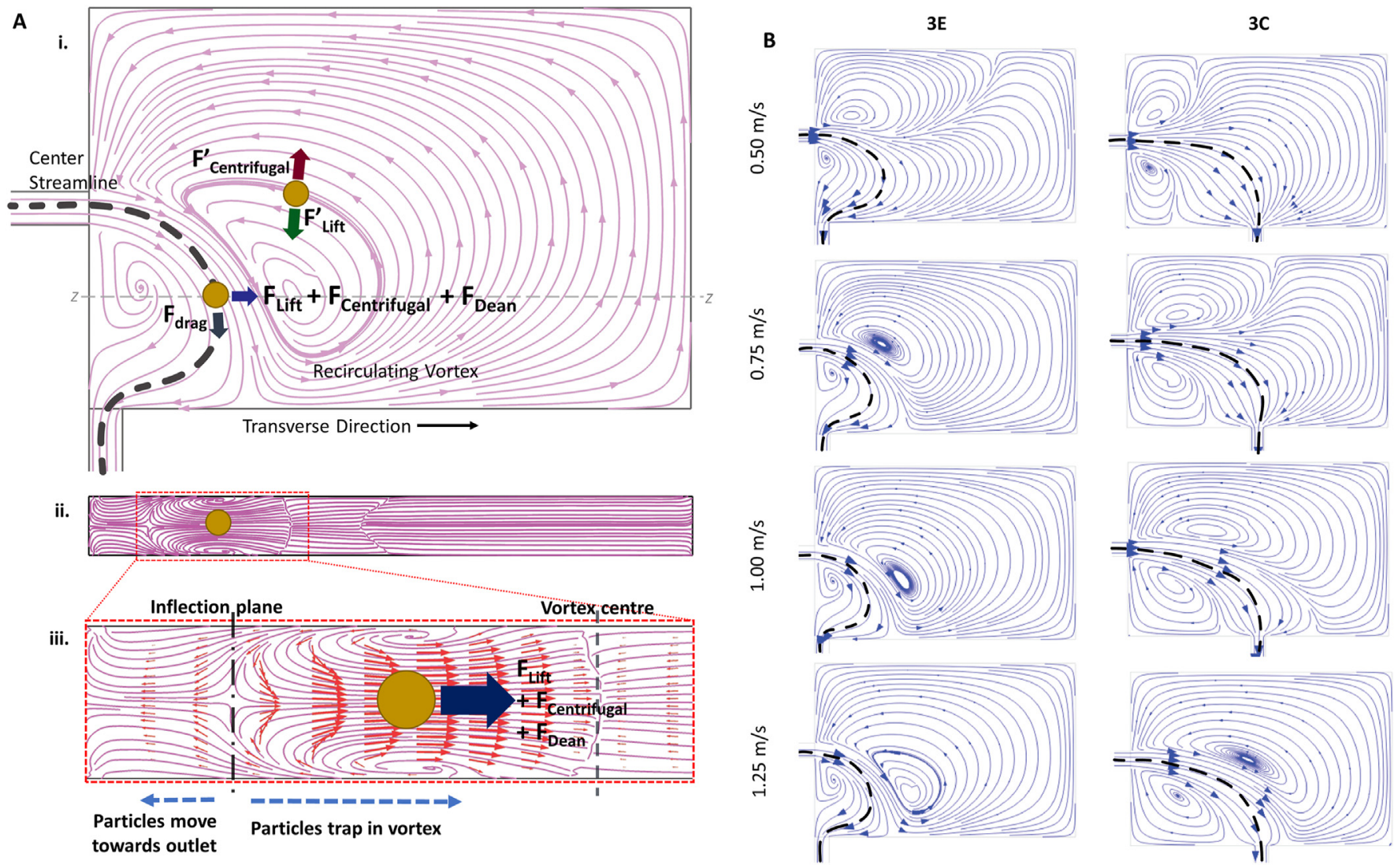

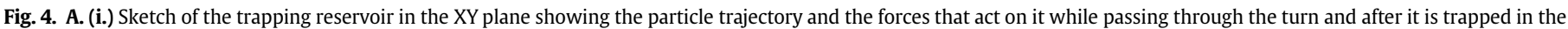

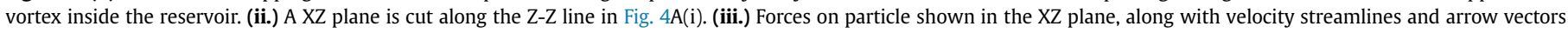

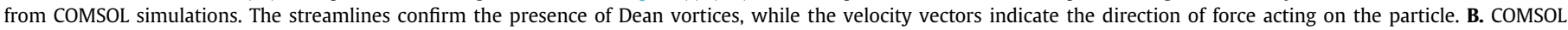
Simulations showing flow streamlines along with approximate center streamline in designs $3 \mathrm{E}$ and $3 \mathrm{C}$ at different velocities comparing the vortex sizes and $r_{\text {turn }}$.

particles are thus expected to migrate laterally out of the centerline flow stream and into the vortex, while smaller particles below a critical size will not migrate fast enough to cross into the vortex before passing out of the reservoir. The above estimate of forces also validates the experimental observations in which the chosen devices (designs 3D, 3E, 4D and 4E) work better with stable trapping at low velocities. For all the device configurations, the sheargradient lift force experienced by the particle remains approximately the same. However, with the inlet and outlet closer to each other, $r_{\text {turn }}$ is reduced (see Fig. $4 \mathrm{~B}$ ) and hence there is a larger Dean drag and centrifugal force directing the particle towards the vortex.

We can estimate the scaling of trapping velocities for different particle sizes. The particle of size $a$ experiences a transverse migration force $F_{T} \propto a^{3} U_{f}^{2}$. Here, $U_{f}$ is the flow velocity. The transverse force leads to lateral displacement of the particle towards the vortex trap. The transverse Stokes drag provides the scaling for the lateral velocity of the particle as $a^{3} U_{f}^{2} \propto \eta a U_{t}$. Here, $\eta$ is fluid viscosity and $U_{t}$ is the transverse velocity. For a given flow velocity $U_{f}$, the time the particle spends in the trapping chamber is given by $t_{c} \approx L / U_{f}$ (where $L$ is the length scale of the path in the chamber). Based on our simulation results, we assume that the path length $(L)$ remains within the same order for velocity regimes of interest. We assume that for trapping, the transverse distance covered to get trapped $\left(x_{a} \approx t_{c} U_{t}\right)$ should be equal for all particles. Using the above equation, we get $x_{a} \propto L a^{2} U_{f} / 6 \pi \eta$. Using this scaling, we can estimate the approximate velocity required for trapping particles of various sizes when the trapping velocity for a particle of a different size is known $\left(a_{1}^{2} U_{f, 1}=a_{2}^{2} U_{f, 2}\right)$.
Furthermore, once a particle has entered the vortex, its orbit is determined by a balance [45] of the centrifugal force, shear gradient lift force, and drag forces due to secondary flows in the transverse direction (Fig. 4A(i)). Acting in the direction of decaying shear gradient, the shear-gradient lift force is still operational in the vortex leading to a force towards the vortex center. Centrifugal force arising from the closed recirculating trajectory of the particle is directed outwards. Even though devices with inlet and outlet in immediate vicinity (designs 5D and $5 \mathrm{E}$ ) result in smaller trapping velocities, they show unsteady trapping (see Fig. S3(A)). These designs have smaller trapping orbitals close to the primary flow. Smaller trapping orbital means that at equilibrium both centrifugal and lift forces are large, and also hints at steric crowding for the trapped particles. Thus, even a small change in the trapping-orbital due to fluctuations in flow velocity or disturbance due to crowding results in unstable and irregular trapping.

The previously reported linear vortex-chip depended only on the shear-gradient lift force in order to accomplish size-specific cell and particle isolation, and hence required high flow velocities in order to generate significant lift force. Notably, the incorporation of turn effect gives rise to a number of additional forces which collectively enable vortex trapping at flow velocities $(1.2-1.3 \mathrm{~m} / \mathrm{s})$ that are significantly lesser compared to those of linear vortexgenerating architectures.

\subsection{Particle Stokes number}

Under the influence of forces associated with the turn effect, trapping also depends on the time spent by the particle inside the 

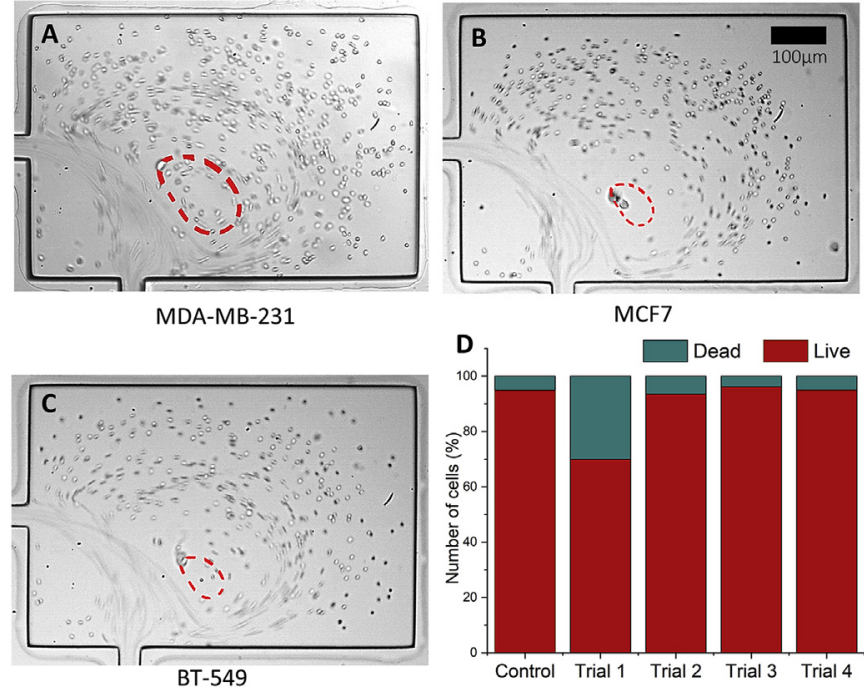

Fig. 5. A-C Trajectory of 3 types of cancer cells (MDA-MB-231, MCF-7 and BT-549) suspended in whole blood when flowed through the orthogonal chip. Scale bar represents $100 \mu \mathrm{m}$. D. Mean Viability of MDA-MB-231 in the device with 4 channels and 6 reservoirs in each was $88.67 \%$ with a standard deviation of $10.82 \%$.

reservoir and whether this time is enough for the transverse forces to sufficiently modify the particle's trajectory. This behavior of particles in the fluid flow can be explained by considering the particle Stokes number St. St is defined as the ratio of the inertial relaxation time $\left(\tau_{\gamma}\right)$ of the particle to the characteristic time of flow $\left(\tau_{f}\right)[55]:$

$S t=\frac{\tau_{\gamma}}{\tau_{f}}=\frac{\rho_{p} a^{2} / 18 \eta}{D_{h} / U}=\frac{\beta a^{2} U}{18 v D_{h}}$

where $\beta$ is the ratio of particle density $\left(\rho_{P}\right)$ to fluid density $\left(\rho_{f}\right)$, and $\nu$ is the kinematic viscosity. A larger St implies that the particle has a stronger preference to persist with its original velocity direction instead of following the accelerating fluid trajectory [55]. St here thus serves to be an indirect measure of the particle inertia and describes the end effect. The cells and particles eject out differentially upon introducing the abrupt orthogonal turn in the flow owing to their size dependent inertia. Larger particles $(20 \mu \mathrm{m}$ beads, $S t \approx 0.5$ ) bearing larger St shoot through the flow-steam and transiting ahead get trapped in the vortex. Smaller particles (RBCs, $S t \approx 0.05)$ meanwhile continue flowing along the stream or circulate in the unsteady outer zone and pass-by undisturbed (see Fig. S7). However, it is important to note here that while St offers a reasonable generic explanation of the phenomenon and can be used to estimate particle behavior and trapping-velocity for a given particle diameter, it does not account for all the physical factors involved in the turn effect.

\subsection{Trapping of cancer cells suspended in whole blood}

In order to verify whether cancer cells could also be separated from a suspension in whole blood, we flowed cells from three transformed malignant breast cancer cell lines: MDA-MB-231, MCF-7 and BT-549 (which show mild differences in size and shape), along with whole blood diluted in PBS. The orthogonal configuration in our device was able to effectively isolate cancer cells from other blood cells: while the former were trapped within vortices, the latter flowed out directly or after passing through outer unstable orbitals of the vortex. (Fig. 5(A-C) and See Movie M3-5).

Similar to our experiments with the polystyrene beads, the high-speed camera videos of cell flows showed a constraint on the number of cancer cells that could occupy a given orbital within the vortex, where a few larger rare cells get trapped in a reservoir. Cancer cells entering an already occupied vortex would 'knock out' the existing one or get extruded out themselves. However, this number of trapped cancer cells can be scaled up by connecting multiple reservoirs in series and parallel.

Finally, we also wanted to check if the cells subjected to the abovementioned forces for isolation were viable enough to be cultured for further assays. Experiments to check for the cellular viability using Trypan Blue (which selective stains dead cells) were carried out for MDA-MB-231 cells that were flowed in a device with 4 channels with 6 reservoirs. About $88.67 \%$ cells (with a standard deviation of $10.82 \%$ ) were found to be alive after egressing from the device, implicating the potential compatibility of such isolation architectures for expansion and enrichment of CTCs (Fig. 5D). MDAMB-231 cells viability at $1.3 \mathrm{~m} / \mathrm{s}$ were comparable to cells just before they entered the device. However, when the inlet flow velocity was increased to $4 \mathrm{~m} / \mathrm{s}$ the viability dropped (see Fig. S8(A)). Also, we were able to trap $20 \mu \mathrm{m}$ beads and MDA-MB-231 cells at a concentration of 30 particles/mL which are in the range of CTCs present in human blood.

\section{Conclusion}

An architecture with inlet and outlet placed close to each other in orthogonal arrangement forms larger vortices at lower flow velocities. Owing to the combined interaction of forces arising from the turn-effect, i.e., shear-gradient lift-, centrifugal- and Deanforces acting against an advective fluid drag force, the orthogonal vortex chip is capable of effective vortex trapping even at low flow velocities. This would not only help maintain the viability of cells (see Fig. S8(A)), but also save the need of any special capacity pumps and apparatus that would be required to meet the high pressure flow rates involved in other such devices $[24,32,39,40,44,45]$ including inlet leakages starting at $1.3 \mathrm{~atm}$ inlet pressure (see Fig. S8(B)). The major utilization would be in separating CTCs in a passive and marker-agnostic manner; which would be carried out at high throughput and yet would involve flow velocities that are reasonably low enough to not impair the viability of cells. Using such label-free methods may not ensure high purity (or specificity of enriched cells) but the capture efficiency, which is potentially important for culture of CTCs and CTC-based inferences, would be optimal. Furthermore, the size-specific attractor-ring in the vortex and the 'knocking-out' phenomenon, along with the aspects of viability, purity and efficiency for the devices pertaining to CTC isolation are to be investigated in detail in future efforts.

\section{CRediT authorship contribution statement}

Navya Rastogi: Methodology, Investigation, Validation, Software, Formal analysis, Visualization, Writing - original draft. Pranjal Seth: Conceptualization, Methodology, Investigation, Software, Visualization, Writing - original draft. Ramray Bhat: Methodology, Funding acquisition, Writing - review \& editing, Supervision. Prosenjit Sen: Methodology, Software, Visualization, Funding acquisition, Writing - review \& editing, Supervision. 


\section{Declaration of competing interest}

The authors declare that they have no known competing financial interests or personal relationships that could have appeared to influence the work reported in this paper.

\section{Acknowledgements}

We thank Dharma Pally and Shahid Hussain for help with cell culture and experiments. The devices were fabricated and characterized in the National Nanofabrication Center and Micro Nano Characterization Facility respectively. This work was supported by DBT Nanotechnology Funds. R.B would like to acknowledge funding support from the Department of Biotechnology, [BT/PR26526/GET/ 119/92/2017], SERB [ECR/2015/000280] and Institute of Eminence grant (IE/CARE-19-0319)].

\section{Appendix A. Supplementary data}

Supplementary data to this article can be found online at https://doi.org/10.1016/j.aca.2021.338423.

\section{References}

[1] R. Nasiri, A. Shamloo, S. Ahadian, L. Amirifar, J. Akbari, M.J. Goudie, et al., Microfluidic-based approaches in targeted cell/particle separation based on physical properties: fundamentals and applications, Small 16 (2020) 2000171.

[2] L. Sun, W. Yang, S. Cai, Y. Chen, H. Chu, H. Yu, et al., Recent advances in microfluidic technologies for separation of biological cells, Biomed. Microdevices 22 (2020) 55 .

[3] A. Dalili, E. Samiei, M. Hoorfar, A review of sorting, separation and isolation of cells and microbeads for biomedical applications: microfluidic approaches, Analyst 144 (2019) 87-113.

[4] M. Antfolk, T. Laurell, Continuous flow microfluidic separation and processing of rare cells and bioparticles found in blood - a review, Anal. Chim. Acta 965 (2017) 9-35.

[5] C. Wyatt Shields Iv, C.D. Reyes, G.P. López, Microfluidic cell sorting: a review of the advances in the separation of cells from debulking to rare cell isolation, Lab Chip 15 (2015) 1230-1249.

[6] P. Sajeesh, A.K. Sen, Particle separation and sorting in microfluidic devices: a review, Microfluid. Nanofluidics 17 (2014) 1-52.

[7] A.A.S. Bhagat, H. Bow, H.W. Hou, S.J. Tan, J. Han, C.T. Lim, Microfluidics for cell separation, Med. Biol. Eng. Comput. 48 (2010) 999-1014.

[8] G. Galletti, L. Portella, S.T. Tagawa, B.J. Kirby, P. Giannakakou, D.M. Nanus, Circulating tumor cells in prostate cancer diagnosis and monitoring: an appraisal of clinical potential, Mol. Diagn. Ther. 18 (2014) 389-402.

[9] W. Zheng, Y. Zhang, L. Guo, S. Wang, M. Fang, W. Mao, et al., Evaluation of therapeutic efficacy with CytoSorter(®) circulating tumor cell-capture system in patients with locally advanced head and neck squamous cell carcinoma, Canc. Manag. Res. 11 (2019) 5857-5869.

[10] A. Lucci, C. Hall, S.P. Patel, B. Narendran, J.B. Bauldry, R. Royal, et al., Circulating Tumor Cells and Early Relapse in Node-Positive Melanoma, Clinical Cancer Research, 2020 clincanres.2670.019.

[11] L.M. Millner, M.W. Linder, R. Valdes Jr., Circulating tumor cells: a review of present methods and the need to identify heterogeneous phenotypes, Ann. Clin. Lab. Sci. 43 (2013) 295-304.

[12] M. Yu, A. Bardia, N. Aceto, F. Bersani, M.W. Madden, M.C. Donaldson, et al., Ex vivo culture of circulating breast tumor cells for individualized testing of drug susceptibility, Science 345 (2014) 216-220.

[13] D. Lv, Z. Hu, L. Lu, H. Lu, X. Xu, Three-dimensional cell culture: a powerful tool in tumor research and drug discovery, Oncol Lett 14 (2017) 6999-7010.

[14] B. Çetin, D. Li, Dielectrophoresis in microfluidics technology, Electrophoresis 32 (2011) 2410-2427.

[15] M. Hejazian, W. Li, N.-T. Nguyen, Lab on a chip for continuous-flow magnetic cell separation, Lab Chip 15 (2015) 959-970.

[16] Z. Wang, J. Zhe, Recent advances in particle and droplet manipulation for labon-a-chip devices based on surface acoustic waves, Lab Chip 11 (2011) 1280-1285.

[17] G.E. Fridley, H. Le, P. Yager, Highly sensitive immunoassay based on controlled rehydration of patterned reagents in a 2-dimensional paper network, Anal. Chem. 86 (2014) 6447-6453.

[18] B.J. Kirby, M. Jodari, M.S. Loftus, G. Gakhar, E.D. Pratt, C. Chanel-Vos, et al., Correction: functional characterization of circulating tumor cells with a prostate-cancer-specific microfluidic device, PloS One 7 (2012), https:// doi.org/10.1371/annotation/9d3d22ed-dc5a-4484-9254-1584864f4aac.
[19] G.M. Cann, Z.G. Gulzar, S. Cooper, R. Li, S. Luo, M. Tat, et al., mRNA-seq of single prostate cancer circulating tumor cells reveals recapitulation of gene expression and pathways found in prostate cancer, PloS One 7 (2012), e49144.

[20] S.L. Stott, C.-H. Hsu, D.I. Tsukrov, M. Yu, D.T. Miyamoto, B.A. Waltman, et al., Isolation of circulating tumor cells using a microvortex-generating herringbone-chip, Proc. Natl. Acad. Sci. Unit. States Am. 107 (2010) 18392.

[21] S. Nagrath, L.V. Sequist, S. Maheswaran, D.W. Bell, D. Irimia, L. Ulkus, et al., Isolation of rare circulating tumour cells in cancer patients by microchip technology, Nature 450 (2007) 1235-1239.

[22] M. Cristofanilli, G.T. Budd, M.J. Ellis, A. Stopeck, J. Matera, M.C. Miller, et al., Circulating tumor cells, disease progression, and survival in metastatic breast cancer, N. Engl. J. Med. 351 (2004) 781-791.

[23] M. Zborowski, J.J. Chalmers, Rare cell separation and analysis by magnetic sorting, Anal. Chem. 83 (2011) 8050-8056.

[24] J. Che, V. Yu, M. Dhar, C. Renier, M. Matsumoto, K. Heirich, et al., Classification of large circulating tumor cells isolated with ultra-high throughput microfluidic Vortex technology, Oncotarget 7 (2016).

[25] F. Castro-Giner, N. Aceto, Tracking cancer progression: from circulating tumor cells to metastasis, Genome Med. 12 (2020) 31.

[26] C. Agnoletto, L. Minotti, L. Brulle-Soumare, L. Pasquali, M. Galasso, F. Corrà, et al., Heterogeneous expression of EPCAM in human circulating tumour cells from patient-derived xenografts, Biomarker Research 6 (2018) 31.

[27] M. Yamada, M. Nakashima, M. Seki, Pinched flow Fractionation: continuous size separation of particles utilizing a laminar flow profile in a pinched microchannel, Anal. Chem. 76 (2004) 5465-5471.

[28] A.A.S. Bhagat, S.S. Kuntaegowdanahalli, I. Papautsky, Continuous particle separation in spiral microchannels using dean flows and differential migration, Lab Chip 8 (2008) 1906-1914.

[29] L.R. Huang, E.C. Cox, R.H. Austin, J.C. Sturm, Continuous particle separation through deterministic lateral displacement, Science 304 (2004) 987-990.

[30] D.L. Adams, R.K. Alpaugh, S.S. Martin, M. Charpentier, S. Chumsri, M. Cristofanilli, et al., Precision microfilters as an all in one system for multiplex analysis of circulating tumor cells, RSC Adv. 6 (2016) 6405-6414.

[31] M.E. Warkiani, B.L. Khoo, L. Wu, A.K.P. Tay, A.A.S. Bhagat, J. Han, et al., Ultrafast, label-free isolation of circulating tumor cells from blood using spiral microfluidics, Nat. Protoc. 11 (2015) 134.

[32] S.C. Hur, A.J. Mach, D. Di Carlo, High-throughput size-based rare cell enrichment using microscale vortices, Biomicrofluidics 5 (2011) 22206.

[33] J.-S. Park, H.-I. Jung, Multiorifice flow fractionation: continuous size-based separation of microspheres using a series of contraction/expansion microchannels, Anal. Chem. 81 (2009) 8280-8288.

[34] S. Yan, J. Zhang, D. Yuan, W. Li, Hybrid microfluidics combined with active and passive approaches for continuous cell separation, Electrophoresis 38 (2017) 238-249.

[35] M.E. Warkiani, B.L. Khoo, D.S.-W. Tan, A.A.S. Bhagat, W.-T. Lim, Y.S. Yap, et al., An ultra-high-throughput spiral microfluidic biochip for the enrichment of circulating tumor cells, Analyst 139 (2014) 3245-3255.

[36] B. Hong, Y. Zu, Detecting circulating tumor cells: current challenges and new trends, Theranostics 3 (2013) 377-394.

[37] S. Taneda, Visualization of separating Stokes flows, J. Phys. Soc. Jpn. 46 (1979) 1935-1942.

[38] H.K. Moffatt, Viscous and resistive eddies near a sharp corner, J. Fluid Mech. 18 (1964) 1-18.

[39] E. Sollier, D.E. Go, J. Che, D.R. Gossett, S. O’Byrne, W.M. Weaver, et al., Sizeselective collection of circulating tumor cells using Vortex technology, Lab Chip 14 (2014) 63-77.

[40] M. Dhar, J. Wong, A. Karimi, J. Che, C. Renier, M. Matsumoto, et al., High efficiency vortex trapping of circulating tumor cells, Biomicrofluidics 9 (2015), 064116.

[41] D.S. Ketpun A, T. Suwannaphan, S. Bhanpattanakul, A. Pimpin, W. Srituravanich, W. Sripumkhai, W. Jeamsaksiri, P. Piyaviriyakul, The viability of single cancer cells after exposure to hydrodynamic shear stresses in a spiral microchannel: a canine cutaneous mast cell tumor model, Micromachines 9 (2018).

[42] R. Fan, T. Emery, Y. Zhang, Y. Xia, J. Sun, J. Wan, Circulatory shear flow alters the viability and proliferation of circulating colon cancer cells, Sci. Rep. 6 (2016) 27073.

[43] J. Hua, L.E. Erickson, T.-Y. Yiin, L.A. Glasgow, A review of the effects of shear and interfacial phenomena on cell viability, Crit. Rev. Biotechnol. 13 (1993) 305-328.

[44] R. Khojah, R. Stoutamore, D. Di Carlo, Size-tunable microvortex capture of rare cells, Lab Chip 17 (2017) 2542-2549.

[45] A.J. Mach, J.H. Kim, A. Arshi, S.C. Hur, D. Di Carlo, Automated cellular sample preparation using a Centrifuge-on-a-Chip, Lab Chip 11 (2011) 2827-2834.

[46] H. Amini, W. Lee, D. Di Carlo, Inertial microfluidic physics, Lab Chip 14 (2014) 2739-2761.

[47] D. Di Carlo, Inertial microfluidics, Lab Chip 9 (2009) 3038-3046.

[48] D. Stoecklein, D. Di Carlo, Nonlinear microfluidics, Anal. Chem. 91 (2019) 296-314.

[49] D. Di Carlo, D. Irimia, R.G. Tompkins, M. Toner, Continuous inertial focusing, ordering, and separation of particles in microchannels, Proc. Natl. Acad. Sci. Unit. States Am. 104 (2007) 18892-18897. 
[50] D. Di Carlo, J.F. Edd, K.J. Humphry, H.A. Stone, M. Toner, Particle segregation and dynamics in confined flows, Phys. Rev. Lett. 102 (2009), 094503.

[51] D.S.W. Lim, J.P. Shelby, J.S. Kuo, D.T. Chiu, Dynamic formation of ring-shaped patterns of colloidal particles in microfluidic systems, Appl. Phys. Lett. 83 (2003) 1145-1147.

[52] E.W.M. Kemna, R.M. Schoeman, F. Wolbers, I. Vermes, D.A. Weitz, A. van den Berg, High-yield cell ordering and deterministic cell-in-droplet encapsulation using Dean flow in a curved microchannel, Lab Chip 12 (2012) 2881-2887.
[53] S.A. Berger, a. L Talbot, L.S. Yao, Flow in curved pipes, Annu. Rev. Fluid Mech. 15 (1983) 461-512.

[54] A.A.S. Bhagat, S.S. Kuntaegowdanahalli, N. Kaval, C.J. Seliskar, I. Papautsky, Inertial microfluidics for sheath-less high-throughput flow cytometry, Biomed. Microdevices 12 (2010) 187-195.

[55] Z. Wu, B. Willing, J. Bjerketorp, J.K. Jansson, K. Hjort, Soft inertial microfluidics for high throughput separation of bacteria from human blood cells, Lab Chip 9 (2009) 1193-1199. 